\title{
Article \\ Hypersensitivity in Molar Incisor Hypomineralization: Superficial Infiltration Treatment
}

\author{
Alberto Murri Dello Diago ${ }^{1, *(\mathbb{C}}$, Milena Cadenaro ${ }^{2}{ }^{\mathbb{C}}$, Rossana Ricchiuto ${ }^{1}$, Federico Banchelli ${ }^{1}$, Enrico Spinas ${ }^{3} \mathbb{D}_{\text {, }}$ \\ Vittorio Checchi ${ }^{1}(\mathbb{D})$ and Luca Giannetti ${ }^{1}$ \\ 1 Department of Surgery, Medicine, Dentistry and Morphological Sciences related to Transplant, Oncology and \\ Regenerative Medicine, University of Modena and Reggio Emilia, 41125 Modena, Italy; \\ rossana.ricchiuto@gmail.com (R.R.); federico.banchelli@unimore.it (F.B.); vittorio.checchi@unimore.it (V.C.); \\ luca.giannetti@unimore.it (L.G.) \\ 2 Institute for Maternal and Child Health-IRCCS "Burlo Garofolo", University of Trieste, 34137 Trieste, Italy; \\ mcadenaro@units.it \\ 3 Department of Surgical Sciences, University of Cagliari, 09124 Cagliari, Italy; enricospinas@tiscali.it \\ * Correspondence: alberto.murridellodiago@gmail.com; Tel.: +393-288-288-121
}

check for

updates

Citation: Murri Dello Diago, A.;

Cadenaro, M.; Ricchiuto, R.; Banchelli,

F.; Spinas, E.; Checchi, V.; Giannetti, L.

Hypersensitivity in Molar Incisor

Hypomineralization: Superficial

Infiltration Treatment. Appl. Sci. 2021,

11, 1823. https://doi.org/10.3390/

app11041823

Academic Editors: Ivana Miletić and Nissan Joseph

Received: 26 January 2021

Accepted: 17 February 2021

Published: 18 February 2021

Publisher's Note: MDPI stays neutral with regard to jurisdictional claims in published maps and institutional affiliations.

Copyright: (c) 2021 by the authors. Licensee MDPI, Basel, Switzerland. This article is an open access article distributed under the terms and conditions of the Creative Commons Attribution (CC BY) license (https:// creativecommons.org/licenses/by/ $4.0 /)$.

\begin{abstract}
To date, there are no standardized protocols available in the literature for hypersensitivity treatment in molar incisor hypomineralization (MIH) patients. The aim of this study was to evaluate the efficacy of erosion-infiltration treatments with resin in children with a strong hypersensitivity and also to develop a minimally invasive diagnostic-therapeutic pathway for young MIH patients. Patients with clinical signs of MIH were enrolled according to international guidelines. A total of 42 patients (8-14 years old) with sensitivity of at least one molar and patients with post eruptive enamel fractures, but without dentin involvement or cavitated carious lesions were selected. A single superficial infiltration treatment with ICON (DMG, Germany) was performed with a modified etching technique. Sensitivity was tested with the Schiff Scale and Wong Baker Face Scale and was repeated at 12 months follow-up. All patients reported lower sensitivity values at the end of the treatment. Significant differences of sensitivity according to the Schiff scale were reported between $\mathrm{T} 0$ and all subsequent follow-ups, $p<0.05$. The treatment of erosion infiltration with ICON resin is a minimally invasive preventive treatment that significantly improves the problem of hypersensitivity in permanent molars with $\mathrm{MIH}$.
\end{abstract}

Keywords: MIH; hypersensitivity; enamel infiltration; hypomineralization

\section{Introduction}

In 2001, Weerheijm proposed the term molar incisor hypomineralization (MIH) to define a qualitative defect of the enamel from a systemic origin, which can affect 1 to all 4 of the permanent molars and can also involve the permanent incisors [1].

The prevalence of different MIH defects varies according to geographical areas, from $2.4 \%$ to $44 \%$ [2-4]. Clinically, tooth enamel affected by $\mathrm{MIH}$ is hypomineralized and opaque with a porous appearance and it is either white, yellow or brown in color [5-7].

In studies conducted with a polarized light microscope [8-11], the enamel defect appeared so porous that immediately after tooth eruption fractures or cracks under the action of masticatory forces (post-eruptive enamel breakdown-PEB) could occur [12].

The different composition of the enamel affected by $\mathrm{MIH}$ and its altered crystalline structure are the cause of the main clinical issues reported by the affected patients. The high hypersensitivity of these elements is therefore the reason for the poor quality of life during the daily oral maintenance procedures and during the consumption of food and cold drinks.

The treatment of teeth affected by MIH should be a minimally invasive procedure that aims to protect, strengthen and preserve dental structure. Numerous therapeutic 
alternatives have been proposed over time for the treatment of MIH-affected teeth [13,14], but the clinical management of these conditions is very demanding and difficult to learn for generic dentists, who often adopt less conservative treatments [15].

The scientific community exclusively proposes non-specific treatments based on fluoride and/or casein mineralizers or desensitizing pastes, in order to reduce hypersensitivity $[16,17]$. However, there are no studies available defining guidelines for this condition.

The aim of this study is to propose a precise and standardized protocol for the improvement of hypersensitivity in patients with MIH through a superficial resin infiltration technique.

\section{Materials and Methods}

This study was approved by the Ethics Committee of the Area Vasta Emilia Nord, Emilia Romagna, Italy (EC Prot. \# 307/2018/OSS/AOUMO).

All patients' parents/legal guardians read and signed an informed consent for participation in the study and authorization for treatment.

Sixty-seven pediatric patients (6 to 14 years old) were selected from the Department of Pediatric Dentistry, University of Modena and Reggio Emilia. The recruitment period lasted 3 months. Patients were assessed by a dental expert in restorative treatments (A.M.D.D.) and subjects who presented visual enamel defects of permanent molars due to $\mathrm{MIH}$, according to the diagnostic criteria proposed by the European Academy of Pediatric Dentistry (EAPD) in 2010 [18], were considered for study inclusion. Patients with sensitivity of at least one of the molars affected by $\mathrm{MIH}$, or with post eruptive enamel fractures, but without dentin involvement or cavitated carious lesions were approached (Figure 1).

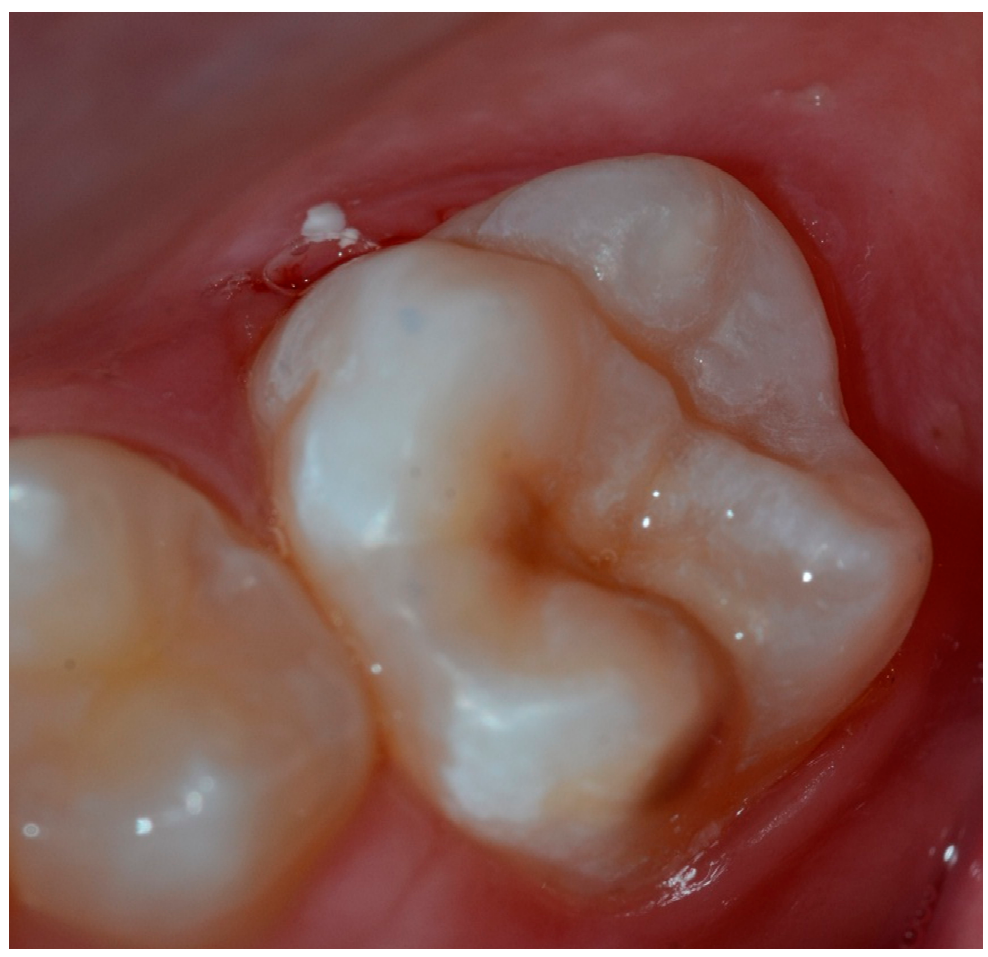

Figure 1. Clinical view of enamel defects of permanent molars due to molar incisor hypomineralization $(\mathrm{MIH})$.

Bitewing examinations were performed to exclude interproximal carious lesions. Twenty-five patients were excluded due to lesions with dentin involvement or cavitated and interproximal caries.

The patient was asked to respond to a questionnaire regarding the selected tooth's sensitivity, both prior to and following the treatment. The patients' parents or legal 
guardians were asked to respond to questions regarding time of gestation, maternal health during gestation, child's weight at delivery, complications during childbirth, diseases in early childhood, breastfeeding, antibiotics assumption during the first two years of life, administration of fluoride-based tablets, and family history of MIH. All information was recorded by an operator and stored in a dedicated database.

Before treatment, the following parameters were recorded into a dedicated Excel spreadsheet: plaque accumulation evaluated by the full mouth plaque score (FMPS) [19], periodontal status with the Löe and Silness gingival index (GI) [20], and bleeding on probing (BoP) with the caton bleeding score [21].

Sensitivity was determined by the examiner using the Schiff cold air sensitivity scale (SCASS), measured by responses to a thermal, cold air jet stimulus $(0=$ subject does not respond to stimulus, 3 = subject responds to stimulus, considers stimulus to be painful, and requests discontinuation of the stimulus) [22]. Sensitivity to tactile stimulation (during oral hygiene and eating ice cream) with patients' response to the intensity of pain was registered according to the Wong-Baker faces scale (WBFS) for pediatric patients $(0=$ no pain, $10=$ intense pain) [23].

All measurements of clinical parameters were repeated at baseline (T0), immediately after treatment (T1), one week (T2), one month (T3), and twelve months (T4) after treatment.

The superficial infiltration technique was performed with ICON (DMG, Hamburg, Germany) $[14,24,25]$, an infiltration system that appears to be extremally useful in the treatment of initial caries and enamel alterations. Manufacturer instructions were followed, with the exception of the etching phase, and carried out with orthophosphoric acid rather than with hydrochloric acid, given the greater porosity of the tissue and the high hypersensitivity of the patient. This technique allows, after an initial erosion phase of the most superficial enamel layer, to infiltrate the porosity system of the defect with a low viscosity resin. The procedure included no anesthesia, rubber dam isolation followed by affected tooth cleaning and removal of any cleaning residue with water spray, $30 \mathrm{~s}$ of etching with $37 \%$ orthophosphoric acid, $30 \mathrm{~s}$ of drying with Icon Dry, 3 min of infiltration with Icon Infiltration, and $40 \mathrm{~s}$ of light curing. The application of Icon Infiltration should be repeated for $1 \mathrm{~min}$, followed by $40 \mathrm{~s}$ of light curing and final polishing.

At each follow-up visit, an objective examination was carried out to assess plaque accumulation and gingival status. The patient was then asked to assign a value from 0 to 10 on the WBFS scale representative of sensitivity, and the operator repeated the Schiff test.

Data were evaluated and expressed as absolute frequencies and percentages, based on the numerical or categorical nature of the variables. Furthermore, a comparative assessment was made between the "post" data and the "pre" data, for each post-intervention timepoint, using statistical inferential tests for paired data (Wilcoxon signed-rank test or similar, after evaluation of the distribution of data), with $95 \%$ confidence level.

\section{Results}

A total of 42 patients were recruited into the study; 22 females and 20 males, aged between 8 and 14 years old. They all presented only one tooth with sensitivity; therefore, there was no need to choose between more than one sensitive tooth to be included into the study. No patients have been lost at the 12 months follow-up recall.

Most of the patients (51\%) had been treated with antibiotic treatment during the first few months of life due to respiratory problems. Moreover, $76 \%$ were born prematurely and/or had problems during childbirth.

Considering the pre-treatment questionnaires completed by the patients' parents/legal guardians, the possible etiological relationship proposed in the literature is confirmed (Table 1). 
Table 1. Pre-treatment questionnaires on etiological causes.

\begin{tabular}{cc}
\hline Etiology Causes & \\
\hline Born prematurely/problem during childbirth & $76 \%$ \\
Fluoride intake & $13 \%$ \\
Asthma & $28.60 \%$ \\
Bronchitis & $33.40 \%$ \\
Pneumonia & $15 \%$ \\
Respiratory tract infections & $31.90 \%$ \\
Celiac disease & $26 \%$ \\
\hline
\end{tabular}

All inflammation indices were not repeated immediately after treatment (T1) as they were not different from T0. The inflammation index drastically reduced following a decreasing trend from the first follow-up. Oral hygiene significantly improved with the absence of plaque in $85.7 \%$ of patients after 12 months of treatment, with a disappearance of gingival inflammation in $81 \%$ of cases. Gingival bleeding persisted only in two patients at all controls, while the remaining 40 showed negative BoP.

The Wong-Baker faces scale during daily hygiene (Figure 2) recorded between 4 and 6 at T0 in $81 \%$ of cases, and between 0 and 2 after 12 months in all patients showing a $100 \%$ improvement.

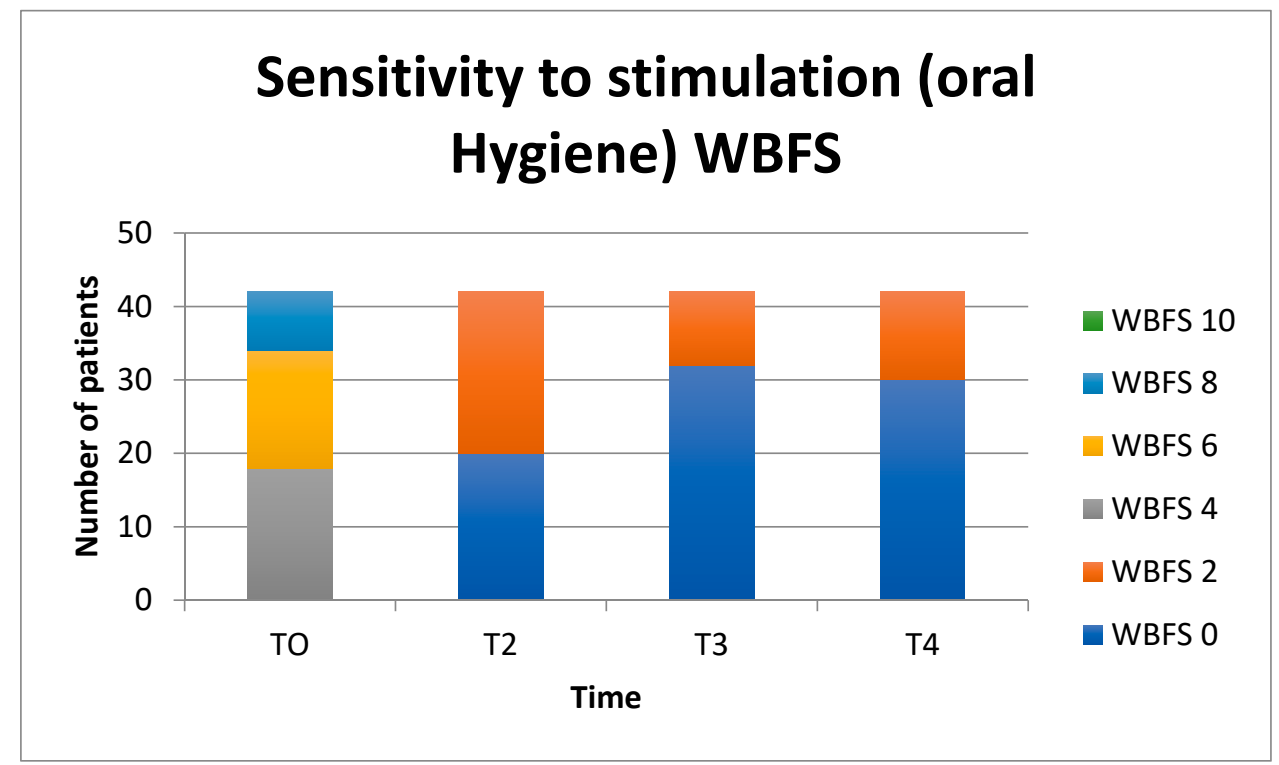

Figure 2. Wong-Baker faces scale (WBFS) sensitivity test to oral hygiene stimulation.

The face scale, referring to the consumption of ice cream (Figure 3), recorded values at T0 of up to 10 in $14 \%$ of cases and 8 in 38\%; at T4 only two children continued to record a value of 4 , while all the others indicated scores of $0-2$, with a $95 \%$ improvement.

Significant differences of sensitivity according to the SCASS scale (Figure 4) were reported between $\mathrm{T} 0$ and all subsequent follow-ups, $p<0.05$. 


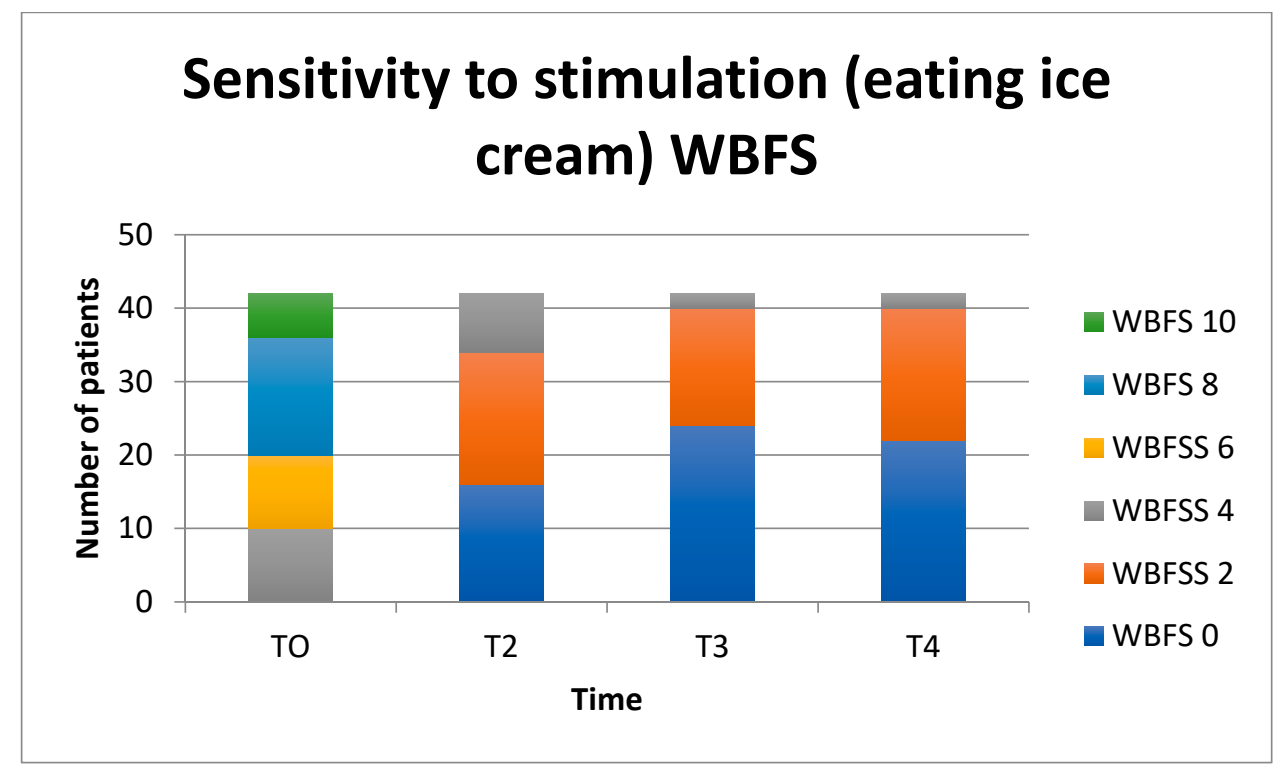

Figure 3. WBFS sensitivity test eating ice cream.

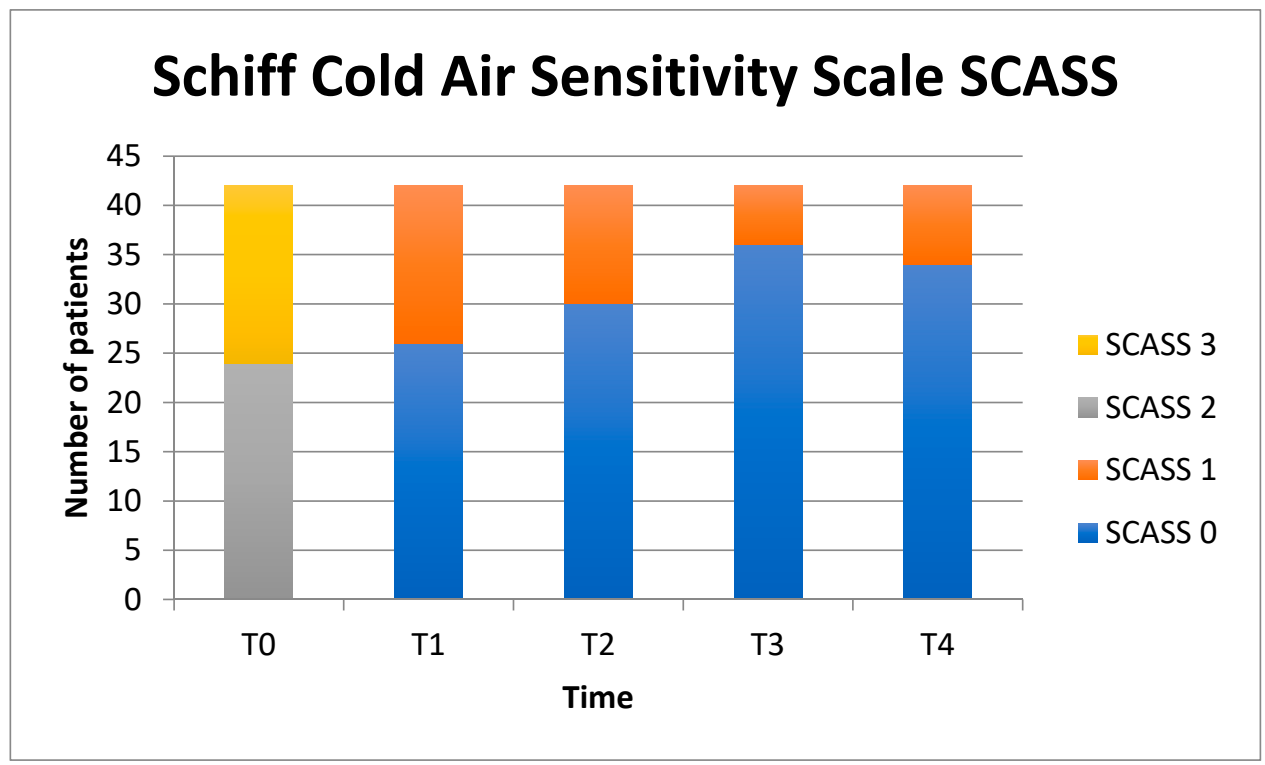

Figure 4. SCASS sensitivity test.

\section{Discussion}

The aim of MIH treatment is to protect, strengthen and preserve the tooth structure when not previously compromised by post eruptive fractures and/or carious lesions. When the pathological scenario is complicated by hypersensitivity to thermal stimuli, the first form of prevention by the clinician is to alleviate the hypersensitivity, in order to facilitate better oral hygiene and reduce the risk of dental caries as much as possible. The choice of preventive treatment is not univocal and the most recent literature examines the use of remineralizers [26]. According to Restrepo, it is evident that the topical application of fluoride varnish does not improve enamel remineralization with MIH and does not consider hypersensitivity [27]. A very recent paper on the use of Casein phosphopeptideamorphous calcium phosphate nanocomplex (CPP-ACP), an optimal remineralizing and anti-cariogen agent, for the treatment of $\mathrm{MIH}$ teeth showed to be effective and safe in bleaching teeth with enamel white opacities but didn't provide significant data regarding hypersensitivity [28]. Ozgul instead published encouraging results on the treatment of hypersensitivity with casein, but with a short follow-up [16]. In a research by Pasini et al., 
a positive follow-up at 120 days was reported in patients with MIH molar hypersensitivity treated with casein, but the described procedure required repeated applications [29].

Recently a new desensitizing paste has been developed, based on Pro-Argin technology, made of $8 \%$ arginine and calcium carbonate for the treatment of dentinal hypersensitivity. Bekes et al. tested sensitivity after 8 weeks of application, but the results did not favor the product with scientific evidence [17]. The data report an improvement of the mechanical characteristics of the enamel of anterior teeth with MIH after the application of a resin infiltration system, suggesting that this technique could be a valid alternative for hypersensitivity to thermal stimuli due to occlusion of porosity [30,31].

The results of this study are important preliminary data to define a new minimally invasive approach protocol for permanent molars with hypersensitivity. This study shows a significant reduction in hypersensitivity in $\mathrm{MIH}$ molars already after one single infiltration with resin. The proposed protocol foresees a single application of resin infiltration, and the reported results do not contradict its effectiveness even in daily home hygiene procedures or during the consumption of cold foods [31].

Sensitivity decreased drastically immediately after the treatment and the improvement trend in the following months suggests a stabilization of the resin within the inter-prismatic gaps, even under the action of the masticatory load.

In all patients, an improvement in the SCASS value was obtained compared to that registered before the treatment. The hypothesis is that having started from very high sensitivity values, they also experience minimal sensory changes that will stabilize over time. In only six patients the stimulus was still perceived after one month, but not as painful as prior to treatment.

Questionnaires of patient treatment evaluation revealed a $100 \%$ patient satisfaction.

\section{Conclusions}

The study conducted, despite the small number of patients, provides useful and statistically significant preliminary data. Larger studies with extended follow-up will be useful for reinforcing these results. Furthermore, this non-invasive treatment could be repeated in patients with persistent sensitivity stimulus, and its efficacy should be investigated.

Author Contributions: Conceptualization, M.C. and L.G.; methodology, A.M.D.D. and R.R.; validation, E.S., V.C. and L.G.; formal analysis, E.S. and A.M.D.D.; investigation, A.M.D.D., R.R. and F.B.; data curation, F.B.; writing-original draft preparation, A.M.D.D., R.R. and F.B.; writing-review and editing, M.C., E.S., V.C. and L.G.; visualization, M.C. and V.C.; supervision, E.S. and L.G.; project administration, A.M.D.D. and L.G. All authors have read and agreed to the published version of the manuscript.

Funding: This research received no external funding.

Institutional Review Board Statement: The study was conducted according to the guidelines of the Declaration of Helsinki, and approved by the Ethics Committee of the Area Vasta Emilia Nord, Emilia Romagna, Italy (EC Prot. \# 307/2018/OSS/AOUMO).

Conflicts of Interest: The authors declare no conflict of interest.

\section{References}

1. Weerhrijm, K. Molar Incisor Hypomineralisation (MIH). Eur. J. Paediatr. Dent. 2003, 3, 114-120.

2. Tirlet, G.; Chabouis, H.F.; Attal, J.P. Infiltration, a new therapy for masking enamel white spots: A 19- month follow-up case series. Eur. J. Esthet. Dent. 2013, 8, 180-190. [PubMed]

3. Steffen, R.; Krämer, N.; Bekes, K. The Würzburg MIH concept: The MIH treatment need index (MIH TNI): A new index to assess and plan treatment in patients with molar incisior hypomineralisation (MIH). Eur. Arch. Paediatr. Dent. 2017, 18, 355-361. [CrossRef] [PubMed]

4. Hartsock, L.A.; Burnheimer, J.; Modesto, A.; Vieira, A.R. A Snapshot of the Prevalence of Molar Incisor Hypomineralization and Fluorosis in Pittsburgh, Pennsylvania, USA. Pediatr. Dent. 2020, 42, 36-40. [PubMed]

5. Jälevik, B. Prevalence and Diagnosis of Molar-Incisor-Hypomineralisation (MIH): A systematic review. Eur. Arch. Paediatr. Dent. 2010, 11, 59-64. [CrossRef] 
6. Jälevik, B.; Norén, J.G. Enamel hypomineralization of permanent first molars: A morphological study and survey of possible aetiological factors. Int. J. Paediatr. Dent. 2000, 10, 278-289. [CrossRef]

7. Apponi, R.; Presti, S.; Spinas, E.; Giannetti, L. Biological genetic and aetiology aspects in molar incisor hypomineralization. J. Biol. Regul. Homeost. Agents 2020, 34, 1219-1222.

8. Da Costa-Silva, C.M.; Ambrosano, G.M.; Jeremias, F.; De Souza, J.F.; Mialhe, F.L. Increase in severity of molar- incisor hypomineralization and its relationship with the colour of enamel opacity: A prospective cohort study. Int. J. Paediatr. Dent. 2011, 21, 333-341. [CrossRef]

9. Denis, M.; Atlan, A.; Vennat, E.; Tirlet, G.; Attal, J.P. White defects on enamel: Diagnosis and anatomopathology: Two essential factors for proper treatment (part 1). Int. Orthod. 2013, 11, 139-165. [CrossRef]

10. Farah, R.A.; Monk, B.C.; Swain, M.V.; Drummond, B.K. Protein content of molar-incisor hypomineralisation enamel. J. Dent. 2010, 38, 591-596. [CrossRef]

11. Crombie, F.A.; Manton, D.J.; Palamara, J.E.; Zalizniak, I.; Cochrane, N.J.; Reynolds, E.C. Characterisation of developmentally hypomineralised human enamel. J. Dent. 2013, 41, 611-618. [CrossRef]

12. Bullio Fragelli, C.M.; Jeremias, F.; Feltrin de Souza, J.; Paschoal, M.A.; de Cássia Loiola Cordeiro, R.; Santos-Pinto, L. Longitudinal Evaluation of the Structural Integrity of Teeth Affected by Molar Incisor Hypomineralisation. Caries Res. 2015, 49, 378-383. [CrossRef]

13. Giannetti, L.; Murri Dello Diago, A.; Corciolani, E.; Spinas, E. Deep infiltration for the treatment of hypomineralized enamel lesions in a patient with molar incisor hypomineralization: A clinical case. J. Biol. Regul. Homeost. Agents 2018, 32, 751-754.

14. Giannetti, L.; Murri Dello Diago, A.; Silingardi, G.; Spinas, E. Superficial infiltration to treat white hypomineralized defects of enamel: Clinical trial with 12-month follow-up. J. Biol. Regul. Homeost. Agents 2018, 32, 1335-1338. [PubMed]

15. Giannetti, L.; Bartoli, G.; Banchelli, F.; Spinas, E.; Murri Dello Diago, A. MIH: A survey amongst dental practitioners in Modena and Reggio Emilia districts. Dent. Cadmos 2018, 86, 172-180. [CrossRef]

16. Ozgül, B.M.; Saat, S.; Sönmez, H.; Oz, F.T. Clinical evaluation of desensitizing treatment for incisor teeth affected by molar-incisor hypomineralization. J. Clin. Pediatr. Dent. 2013, 38, 101-105. [CrossRef] [PubMed]

17. Bekes, K.; Heinzelmann, K.; Lettner, S.; Schaller, H.G. Efficacy of desensitizing products containing $8 \%$ arginine and calcium carbonate for hypersensitivity relief in MIH-affected molars: An 8-week clinical study. Clin. Oral. Investig. 2017, 21, 2311-2317. [CrossRef]

18. Lygidakis, N.A.; Wong, F.; Jälevik, B.; Vierrou, A.M.; Alaluusua, S.; Espelid, I. Best Clinical Practice Guidance for clinicians dealing with children presenting with Molar-Incisor-Hypomineralisation (MIH). Eur. Arch. Paediatr. Dent. 2010, 11, 75-81. [CrossRef] [PubMed]

19. Bentley, C.D.; Disney, J.A. A comparison of partial and full mouth scoring of plaque and gingivitis in oral hygiene studies. J. Clin. Periodontol. 1995, 22, 131-135. [CrossRef]

20. Loe, H.; Silness, J. Periodontal disease in pregnancy I. Prevalence and severity. Acta Odontol. Scand. 1963, 21, 533-551. [CrossRef] [PubMed]

21. O'Leary, T.J.; Drake, R.B.; Naylor, J.E. The plaque control record. J. Periodontol. 1972, 43, 38. [CrossRef]

22. Schiff, T.; Delgado, E.; Zhang, Y.P.; Cummins, D.; De Vizio, W.; Mateo, L.R. Clinical evaluation of the efficacy of an in-office desensitizing paste containing $8 \%$ arginine and calcium carbonate in providing instant and lasting relief of dentin hypersensitivity. Am. J. Dent. 2009, 22, 8a-15a. [PubMed]

23. Wong, D.L.; Baker, C.M. Pain in children: Comparison of assessment scales. Pediatr. Nurs. 1988, 14, 9-17. [PubMed]

24. Attal, J.P.; Atlan, A.; Denis, M.; Vennat, E.; Tirlet, G. White spots on enamel: Treatment protocol by superficial or deep infiltration (part 2). Int. Orthod. 2014, 12, 1-31. [CrossRef]

25. Jia, L.; Stawarczyk, B.; Schmidlin, P.R.; Attin, T.; Wiegand, A. Effect of caries infiltrant application on shear bond strength of different adhesive systems to sound and demineralized enamel. J. Adhes. Dent. 2012, 14, 569-574. [PubMed]

26. Ghanim, A.; Silva, M.J.; Elfrink, M.E.C.; Lygidakis, N.A.; Marino, R.J.; Weerheijm, K.L.; Manton, D.J. Molar incisor hypomineralisation (MIH) training manualfor clinical field surveys and practice. Eur. Arch. Paediatr. Dent. 2017, 18, 225-242. [CrossRef]

27. Restrepo, M.; Jeremias, F.; Santos-Pinto, L.; Cordeiro, R.C.; Zuanon, A.C. Effect of Fluoride Varnish on Enamel Remineralization in Anterior Teeth with Molar Incisor Hypomineralization. J. Clin. Pediatr. Dent. 2016, 40, 207-210. [CrossRef]

28. Mastroberardino, S.; Campus, G.; Strohmenger, L.; Villa, A.; Cagetti, M.G. An Innovative Approach to Treat Incisors Hypomineralization (MIH): A Combined Use of Casein Phosphopeptide- Amorphous Calcium Phosphate and Hydrogen Peroxide-A Case Report. Case Rep. Dent. 2012, 2012, 379593. [CrossRef] [PubMed]

29. Pasini, M.; Giuca, M.R.; Scatena, M.; Gatto, R.; Caruso, S. Molar incisor hypomineralization treatment with casein phosphopeptide and amorphous calcium phosphate in children. Minerva Stomatol. 2018, 67, 20-25. [PubMed]

30. Paris, S.; Schwendicke, F.; Seddig, S.; Müller, W.D.; Dörfer, C.; Meyer-Lueckel, H. Micro-hardness and mineral loss of enamel lesions after infiltration with various resins: Influence of infiltrant composition and application frequency in vitro. J. Dent. 2013, 41, 543-548. [CrossRef]

31. Taher, N.M.; Alkhamis, H.A.; Dowaidi, S.M. The influence of resin infiltration system on enamel microhardness and surface roughness: An in vitro study. Saudi Dent. J. 2012, 24, 79-84. [CrossRef] [PubMed] 